\title{
Increasing signal/acoustic interference ratio in telecommunications audio exchange by adaptive filtering methods
}

\author{
Y A Kropotov ${ }^{1}$, A A Belov ${ }^{1}$ and A Y Prockuryakov ${ }^{1}$ \\ ${ }^{1}$ Murom Institute (branch) "Vladimir State University named after Alexander and Nicholay \\ Stoletovs", Orlovskaya street, 23, Murom, Russia, 602264 \\ e-mail: kaf-eivt@yandex.ru
}

\begin{abstract}
The paper deals with the issues of increasing signal/noise ratio in telecommunication audio exchange systems. The study of characteristics of speech signals and acoustic noises, such as mathematical expectation, dispersion, relative intensity of acoustic speech signals and various types of acoustic noises and interference is carried out. It is shown that in the design of telecommunications systems, in particular loudspeaker systems operating under the influence of external acoustic noise of high intensity, it is necessary to solve the problem of developing algorithms to effectively suppress the above mentioned interference to ensure the necessary signal/noise ratio in communication systems. A mathematical model of the autocorrelation function of the speech signal by using the Lagrange interpolation polynomial of order 10, considered the creation of adaptive algorithms to suppress acoustic noise by linear filtering methods. Thus suppression of acoustic noises and hindrances is possible at the expense of operated change of area of a cutting in the interval from $0 \mathrm{~Hz}$ to 300 $1000 \mathrm{~Hz}$, depending on a hindrance conditions.
\end{abstract}

\section{Introduction}

The issues of increasing signal/acoustic interference in telecommunications audio exchange are relevant and widely covered in the domestic [1-4] and foreign literature [5-8]. As the main criteria for the effectiveness of telecommunications systems, to ensure a reliable exchange of information between the subscribers of the system, we can cite the signal/acoustic noise ratio and syllable intelligibility [1].

Reliable exchange of audio information in communication and telecommunication systems is possible with the provision of signal/external acoustic noise ratio in $P_{s} / P_{n} \geq 20 \mathrm{~dB}$, which, accordingly, will provide the necessary syllabic intelligibility $\mathrm{S} \geq 93 \%$ for the effective, complete exchange of audio data and operational-command information in loudspeaker systems at multifunctional facilities of various purposes. For this reason, it is important to continue research aimed at developing new approaches and algorithms to increase signal-to-acoustic interference ratio by methods of adaptive filtration with the use of controlled change of the resection area. 


\section{Investigation of the characteristics of speech signals and acoustic noise}

The known mathematical model of the function of probability density $P\left(x_{i}\right)$ of speech signals, developed in [9], allows to receive values of mathematical expectation $\bar{x}_{i}$ and dispersion $\sigma^{2}$ of speech signals according to expressions

$$
\bar{x}_{i}=\sum_{i=-2^{n}+1}^{2^{n}-1} x_{i} \cdot P\left(x_{i}\right), \quad \sigma^{2}=\sum_{i=-\left(2^{n}-1\right)}^{2^{n}-1} x_{i}^{2} P\left(x_{i}\right),
$$

where $x_{i}$ is the speech signal samples quantized at $2^{n}$ intervals and normalized relative to the maximum values in the form of $\left| \pm x_{\max }\right|=1 \mathrm{~V}$, then we obtain the values $\bar{x}_{i} \approx 0$ and $\sigma^{2}=0,126$ W/Ohm.

Accordingly, the level of sound pressure intensity $I$ relative to the zero level of intensity $I_{0}=10^{-12} \mathrm{~W} / \mathrm{m}^{2}$ is defined as

$$
\frac{I}{I_{0}}(d B)=10 \lg \frac{k \sigma^{2}}{S} \cdot 10^{12},
$$

where $\quad k$ is the directivity coefficient of a loudspeaker device, in case of radiation in one direction is accepted $k=2 ; S=4 \pi R^{2}$ is a sphere area, $R$ is a sphere radius in meters.

Then the relative intensity of the acoustic speech signal $I_{p}$ calculated by means of expression (2) has the following value

$$
\frac{I_{p}}{I_{0}}(d B)=103 d B
$$

in the case of $\sigma^{2}=0,126 \mathrm{~W}, k=2, R=1 \mathrm{~m}$.

The relative intensities for different acoustic noises and acoustic disturbances are also calculated using the values of mathematical expectation and dispersions of different types of acoustic disturbances determined by formulas (1), in which the mathematical model of the function of probability density of acoustic disturbances is applied [10]. Values of relative intensities for different acoustic signals are presented in Table 1.

Table 1. Relative intensities for different acoustic signals.

\begin{tabular}{cccc}
\hline Type of signal & $P(\mathrm{~W})$ & $\frac{I}{I_{0}}(\mathrm{~dB})$ & $\frac{P_{s}}{P_{n}}(\mathrm{~dB})$ \\
\hline Speech signal & 0.126 & 103 & - \\
Quantum noise & $0.5 \cdot 10^{-5}$ & 59 & 44 \\
Acoustic disturbances & $3.5 \cdot 10^{-4}$ & 85 & 18 \\
The noise of the sea & 0.0687 & 90.4 & 12.6 \\
Wind noise & 0.0953 & 91.8 & 11.2 \\
The noise in the engine room & 0.111 & 92.5 & 10.5 \\
\hline
\end{tabular}

Table 1 shows that in the presence of acoustic noise such as sea noise, wind noise, noise in the engine room $P_{s} / P_{n}$ is in the range of $10,5 \div 18 \mathrm{~dB}$. At such signal/interference relations, the syllable intelligibility can be reduced to $65 \%$ for the case of Russian speech transmission. Reliable reception of the transmitted speech information by the subscriber under such conditions is significantly complicated.

According to the research in [10], for the correct reception of the transmitted voice message through a noisy channel, it is necessary to provide a ratio $P_{s} / P_{n}$ of at least $20 \mathrm{~dB}$. Therefore, in the design of telecommunications systems, in particular, hands free systems operating under the influence of external acoustic noise, the task is to create algorithms to effectively suppress the above mentioned interference to ensure the necessary ratio of $P_{s} / P_{n} \geq 20 \mathrm{~dB}$.

Studies of the spectral functions of speech signals and external noise interference in [9] have shown that the spectrum of the most common interference - external acoustic noise - is shifted relative to the 
spectrum of speech signals in the low-frequency region, which suggests that increasing the ratio $P_{s} / P_{n}$ can be applied linear filtering methods. Another factor allowing to apply linear filtration to suppress acoustic noise is the possibility of increasing the cutting area in the range from $F=0 \mathrm{~Hz}$ to $F_{l}=300$ $1000 \mathrm{~Hz}$, according to studies in [10]. From these studies it can be seen that with the increase of the lower boundary of the reproduced frequencies up to $F=1000 \mathrm{~Hz}$ the value of $S$ practically does not change, it takes the value not less than $94 \%$ which is acceptable.

Thus, the above factors allow to consider the possibility of designing adaptive algorithms of speech signal processing and suppression of acoustic interference due to controlled change of the cutting area in the range from $0 \mathrm{~Hz}$ to $(300 \div 1000) \mathrm{Hz}$, depending on the interference situation.

\section{Development of an adaptive filtering algorithm with control of the resection area}

Consider as a model of adaptive filtering a bandpass filter of rectangular shape with a floating lowfrequency cut $F_{l}$ in the AFC channel. Varying $F_{l}$ within $0 \leq F_{l} \leq F_{h}$, where $F_{h}$ is a fixed high-frequency section of the AFC filter. The AFC of the bandpass filter $K(f)$ is specified as

$$
K(f)=\mid \begin{array}{ll}
1, & \text { at } F_{l} \leq f \leq F_{h} \\
0, & \text { at other values } f
\end{array}
$$

where $0 \leq F_{l} \leq F_{h}, F_{h}=6000 \mathrm{~Hz}$.

With the known spectral function of the speech signal $G(f), P_{s}$ at the bandpass filter output is defined as

$$
P_{s}=\int_{F_{l}}^{F_{h}} K^{2}(f) G(f) d f=\int_{F_{l}}^{F_{h}} G(f) d f,
$$

where the second part of the $P_{s}$ calculation expression is rewritten according to the $K^{2}(f)=1$ integration limits of $F_{l}$ to $F_{h}$.

Correspondingly changing $F_{l}$ within the range of $0 \leq F_{l} \leq F_{h}$, we obtain the dependence of $P_{s}$ on the value of low-frequency $F_{l}$ AFC of the channel. Write down the spectral function of the energy spectrum of the speech signal in the form

$$
G(\Delta f \cdot g)=\sum_{g=0}^{N-1} \sum_{k=0}^{k-1} R(k T) e^{-j 2 \pi \Delta f \cdot g \cdot k T},
$$

where $\Delta f=\frac{F d}{N}$ is the sampling interval of the spectral function by frequency, $N$ is number of speech signal samples at the final interval, $g$ is the number of the frequency discrete component of the spectral function reference is within $0 \leq g \leq N-1, R(k T)$ is the voice signal ACF is represented as a grid function in Table 2 for one of the voice signal implementations.

Table 2. Grid function of the voice signal ACF.

\begin{tabular}{lllllll}
\hline$k_{h}, k_{l}$ & $k_{0}=0$ & $k_{1}=7$ & $k_{2}=25$ & $k_{3}=54$ & $k_{4}=93$ & $k_{5}=130$ \\
\hline$R\left(k_{h}\right)$ & $R\left(k_{0}\right)=0,126$ & $R\left(k_{1}\right)=0,115$ & $R\left(k_{2}\right)=0,037$ & $R\left(k_{3}\right)=-0,048$ & $R\left(k_{4}\right)=-0,016$ & $R\left(k_{5}\right)=-0,025$ \\
$k_{h}, k_{l}$ & $k_{6}=182$ & $k_{7}=182$ & $k_{8}=228$ & $k_{9}=245$ & $k_{10}=253$ & \\
$R\left(k_{h}\right)$ & $R\left(k_{6}\right)=0,026$ & $R\left(k_{7}\right)=0,008$ & $R\left(k_{8}\right)=-0,003$ & $R\left(k_{9}\right)=-0,001$ & $R\left(k_{10}\right)=0$ & \\
\hline
\end{tabular}

Mathematical model of speech signal ACF is represented by approximation of interpolation polynomial Lagrangian of the tenth order in the form of

$$
R(k)=\sum_{k=0}^{H=10} R\left(k_{h}\right) \prod_{\substack{r=0 \\ r \neq q}}^{H=10} \frac{k-k_{r}}{k_{q}-k_{r}},
$$

where $k$ is the current delay multiple of the sampling period $T, k_{q}$ is the delay in the node with the number $q, r$ is current delays of other nodes at $r \neq q$.

Similarly, the power of acoustic noise $P_{n}$ is calculated as 


$$
P_{n}=\int_{F_{l}}^{F_{h}} G_{\Pi I}(f) d f .
$$

The spectral function of acoustic noise $G_{I I}(\Delta f \cdot g)$ looks like

$$
G_{\Pi}(\Delta f \cdot g)=\sum_{g=0}^{N-1} \sum_{K=0}^{K-1} R_{\Pi}(k T) l^{-j 2 \pi \Delta f \cdot g \cdot k T},
$$

where $R_{\mathrm{n}}(k T)$ is ACF interference is also represented by its approximation by an interpolation Lagrangian polynomial.

The process of filtration of the spectral functions of the speech signal $G(f)$ and interference $G_{\mathrm{n}}(f)$ and, accordingly, the change in the ratio $P_{s} / P_{n}$ depending on the change in $F_{l}$ within $300 \mathrm{~Hz} \leq F_{l} \leq 1000 \mathrm{~Hz}$ is experimentally simulated and the results are illustrated in Figure 1. Figure 1 clearly shows that the area of the integrative function of $G_{\text {п }}(f)$ corresponding to the $P_{n}$ decreases faster with changes in $F_{l}$ from $300 \mathrm{~Hz}$ to $1000 \mathrm{~Hz}$ than the area of the integrative function of $G(f)$. Figure 1 also demonstrates that the basic energy of the spectral function $G(f)$ remains in the range from $0 \mathrm{~Hz}$ to $F_{l}$, outside the bandwidth of the rectangular filter.

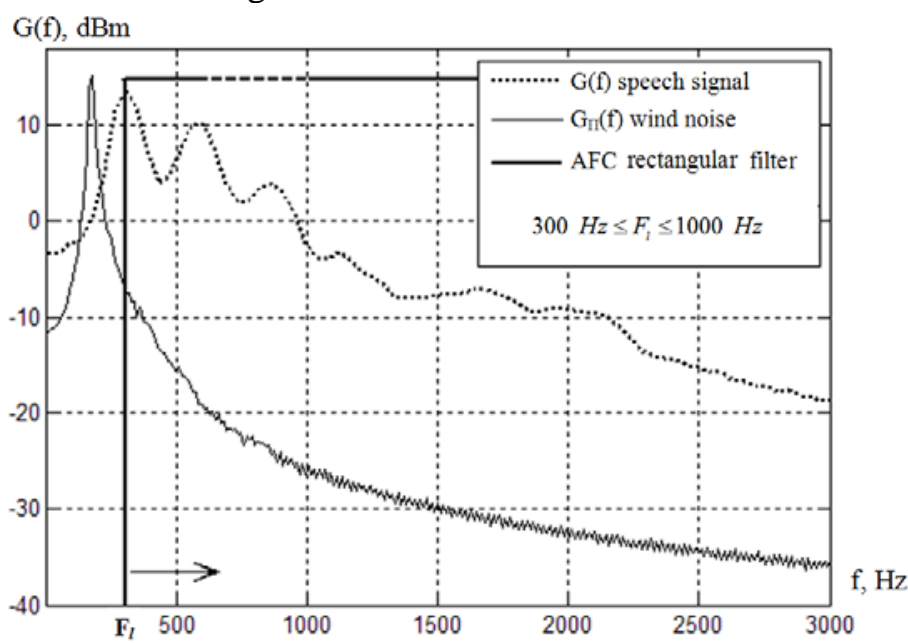

Figure 1. Illustration of the model of filtration of the spectral functions of the speech signal $G(f)$ and the interference $G_{\mathrm{n}}(f)$ depending on the change in the value of $F_{l}$.

Thus, the ratio of the values of $P_{s}$ and $P_{n}$ at the output of the rectangular filter shows the dependence of the degree of suppression of acoustic noise relative to the speech signals on the value of the resection region from $0 \mathrm{~Hz}$ to $F_{l}$ in the AFC. In the discrete case of representation of spectral functions, we obtain the ratio $P_{s} / P_{n}$ in the form of

$$
\frac{P_{s}}{P_{n}}=\frac{\sum_{g_{l}}^{g_{h}} G(g \cdot \Delta f)}{\sum_{g_{l}}^{g_{h}} G_{\Pi}(g \cdot \Delta f)},
$$

where at duration of the analysis interval $\tau_{\text {segm }}$, the number of samples in the analysis interval $N=\frac{\tau_{\text {segm }}}{T}, \Delta f=\frac{F_{d}}{N}$ of the spectral function sampling interval by frequency, $g_{l}=\frac{F_{l}}{\Delta f}$ is the number of frequency interval for low-frequency section of AFC $F_{l}$.

Thus, changing $g_{l}$ in the ratio expression $P_{s} / P_{n}$ will get a function of changing this ratio at the output of the rectangular filter depending on the width of the suppression area, which is in the range from 0 to $F_{l}=g_{1} \Delta f$. 
The obtained results of studies of the effect of change in $F_{l}$ on $P_{s} / P_{n}$ a fixed $F_{h}=6000 \mathrm{~Hz}$ are shown in Figure 2. In researches cases for three various realizations of speech signals concerning various external noise hindrances are considered.

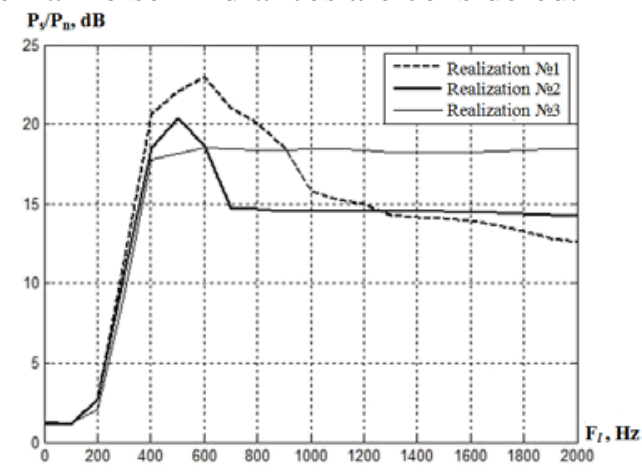

a) Wind noise

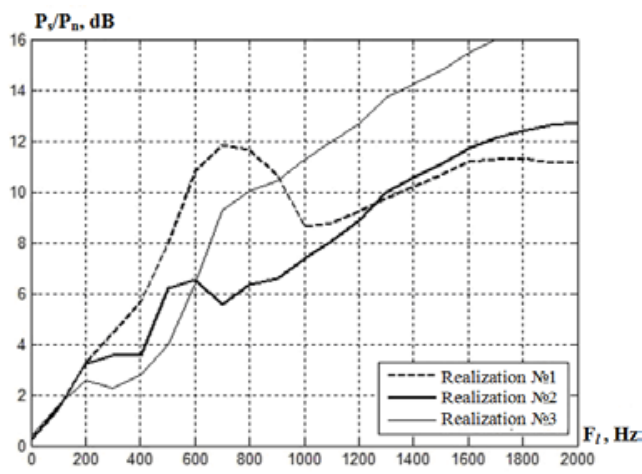

b) The noise in the engine room

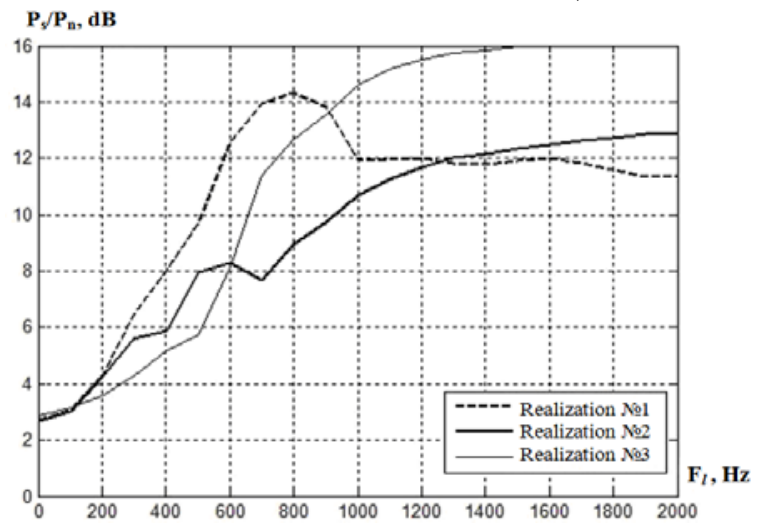

c) The noise of the sea

Figure 2. Dependence of the ratio $P_{s} / P_{n}$ at the output of a rectangular filter on the value of $F_{l}$, at a fixed value of $F_{h}=6000 \mathrm{~Hz}$.

\section{Conclusion}

From the diagrams in Figure 2 it can be seen that external acoustic interference of the "wind noise" type, if set to $F_{l}=500 \mathrm{~Hz}$, is suppressed by $-(17 \div 23) \mathrm{dB}$. At the influence of acoustic noise of the type "noise in the engine room" and at $F_{l}=700 \div 1000 \mathrm{~Hz}$, such acoustic noise is suppressed up to $-12 \mathrm{~dB}$. At the influence of acoustic interference of "sea noise" type at $F_{l}=800 \div 1000 \mathrm{~Hz}$, this interference is suppressed by $(11 \div 15) \mathrm{dB}[10]$. The obtained results of acoustic noise suppression studies show that the linear filtration method can provide the necessary ratio of $P_{s} / P_{n} \geq 20 \mathrm{~dB}$ and, accordingly, the necessary syllabic legibility of $S \geq 93 \%$ in the telecommunications system of voice information exchange.

\section{References}

[1] Zheleznyak V K, Makarov Y K and Khoreev A A 2000 Some methodical approaches toevaluation of efficiency of speech information protection Special technique 4 39-45

[2] Sapozhkov M A 1962 Speech signal in cybernetics and communications (Moscow: Svyazizdat) p 452

[3] Martin R, Heute U and Antweiler C 2008 Advances in Digital Speech Transmission (London: John Wiley and Son) p 572

[4] Ryabenkyi V S 2012 Mathematical model of the external noise suppression devices in the subarea of space Mathematical modeling 24(8) 3-31

[5] McAulay R and Malpass M 1980 Speech enhancement using a soft-decision noise suppression filter IEEE Trans, on Acoustics, Speech, and Signal Processing 28(2) 137-145 
[6] Cohen I, Benesty J and Gannot S 2010 Speech processing in modern communication (Berlin, Heidelberg: Springer) p 342

[7] Hansler E and Schmidt G 2006 Topics in acoustic echo and noise control: Selected methods for the cancelation of acoustic echoes, the reduction of background noise, and speech processing (Berlin, Heidelberg: Springer) p 642

[8] Kahrs M and Brandenburg K 2002 Applications of digital signal processing to audio and acoustics (New York: Kluwer Academic Publisher) p 572

[9] Kropotov Y A and Belov A A 2016 Application method of barrier functions in the problem of estimating the probability density of the parameterized approximations 13th International Scientific-Technical Conference on Actual Problems of Electronic Instrument Engineering 6972

[10] Kolpakov A A and Kropotov Y A 2017 Advanced mixing audio streams for heterogeneous computer systems in telecommunications CEUR Workshop Proceedings 1902 32-36

[11] Kropotov Y A and Proskuryakov A Y 2007 Mathematical model of probability law for the amplitudes of speech waveforms in the exponential basises 17th International Crimean Conference - Microwave and Telecommunication Technology 364-366

[12] Breining C 1999 Acoustic echo control. An application of very-high-order adaptive filters Signal Processing Magazine 16(4) 42-69

[13] Boll S 1979 Suppression of acoustic noise in speech using spectral subtraction IEEE Transactions on Acoustics, Speech, and Signal Processing 27(2) 113-120

[14] Diniz P S 2008 Adaptive filtering algoriths and practical implementation (New York: Springer) p 627

[15] Hakansson L 2002 Noise Reduction in Speech Applications (Boca Raton, Florida: CRC press) p 384

[16] Kropotov Y A, Belov A A and Proskuryakov A Y 2018 Method for forecasting changes in time series parameters in digital information management systems Computer Optics 42(6) 10931100 DOI: 10.18287/2412-6179-2018-42-6-1093-1100 